\title{
Molecular tools to support metabolic and immune function research in the Guinea Fowl (Numida meleagris)
}

\author{
Carl E Darris ${ }^{1}$, James E Tyus ${ }^{1}$, Gary Kelley ${ }^{1}$, Alexander J Ropelewski ${ }^{2}$, Hugh B Nicholas $\mathrm{Jr}^{2}$, Xiaofei Wang ${ }^{1}$ \\ and Samuel Nahashon ${ }^{1 *}$
}

\begin{abstract}
Background: Guinea fowl (Numidia meleagris) production as an alternative source of meat and poultry has shown potential for economic viability. However, there has been little progress in characterizing the transcriptome of the guinea fowl. In this study RNA-sequencing and de novo transcriptome assembly of several Guinea fowl tissues (pancreas, hypothalamus, liver, bone marrow and bursa) which play key roles in regulating feed intake, satiety, and immune function was performed using Illumina's Hi-Seq 2000.
\end{abstract}

Results: 74 million sequences were generated and assembled into 96,492 contigs using the Trinity software suite. Over 39,000 of these transcripts were found to have in silico translated protein sequences that are homologous to chicken protein sequences. Gene ontology analysis uncovered 416 transcripts with metabolic functions and 703 with immune function.

Conclusion: The transcriptome information presented here will support the development of molecular approaches to improve production efficiency of the guinea fowl and other avian species.

Keywords: Guinea fowl, RNA-seq, De novo assembly, Metabolic function, Immune function

\section{Background}

Population projections and high production costs necessitate research to identify and develop alternative sources of meat and poultry. The guinea fowl (GF) is a provocative poultry alternative due to its superior nutritional value and economic potential [1]. Over the past decade poultry research has benefited greatly from advances in sequencing technology, with the genome and various transcriptome projects of both the chicken and the turkey being completed. While there are efforts to generate similar data in non-model avian species [2-4], to date there is very limited genetic information available to aid the effort of bringing GF meat and poultry products into mainstream consumption at a reasonable price [5-17]. The application of transcriptome data generated through RNA-sequencing has the potential to provide clues that

\footnotetext{
* Correspondence: snahashon@tnstate.edu

${ }^{1}$ College of Agriculture, Human and Natural Sciences, Tennessee State University, Nashville, Tennessee, USA

Full list of author information is available at the end of the article
}

will increase our understanding of the metabolic regulation of appetite, feed utilization, immune function, growth and overall production performance of guinea fowl.

Transcriptome analysis of the guinea fowl will provide fundamental data needed to develop species-specific management tools, such as feed and disease prevention regimens. The coupled selection of metabolic and immune function traits is of the utmost importance, as continual selection for single metabolic or growth traits have had a detrimental effect on immune function in poultry [18]. At first glance this may seem a bit surprising because a rapid growth rate is normally associated with good overall health, to include immune function. It has been shown however, that trait selection imposes an energy trade-off within organisms [18]. Previous studies have investigated the trade-offs between immune function versus reproduction, production traits, and growth in birds, sheep, and insects respectively [19-21]. Collectively these studies have shown that immune function as a trait is dynamic, energetically costly and requires optimization in concert with other 
selected traits. Interestingly, van der Most et al. found that while the selection for growth was detrimental to immune function, the selection of immune function does not comprise growth performance in poultry [18]. These findings open the door for the establishment of highperformance lines of poultry by allowing for the selection of disease resistance and growth simultaneously.

Attaining these goals requires a wealth of genetic information and an in-depth understanding of the role played by each gene involved in the regulation of metabolism, satiety, feed utilization, conversion and metabolism, and immune function. De-novo sequencing and transcriptome assembly of the guinea fowl pancreas, hypothalamus, liver, bone marrow and bursa was performed as a first step to developing this required pool of data unique to the GF. Such information is essential in revealing new metabolic pathways that may be utilized to improve growth and production performance of both traditional and non-traditional poultry such as chickens and guinea fowl, respectively.

The aim of this study was to (i) perform targeted de novo assembly of the guinea fowl transcriptome of the pancreas, liver, hypothalamus, spleen, bursa and bone marrow; (ii) to compile a database of functional annotations for the assembled guinea fowl transcriptome; (iii) to perform comparative analysis of the assembled guinea fowl transcriptome using chicken and turkey protein databases; (iv) to identify guinea fowl transcripts with metabolic and immune function.

\section{Results and discussion}

In this study we set out to develop a transcriptome library that would reveal unique gene sequences to aid the understanding of key and unique metabolic and immune processes in the guinea fowl. Application of the data generated in this study will serve to improve the production performance of guinea fowl and other related avian species.

\section{Transcriptome assembly}

As mentioned above, the original 74 million Illumina reads (4.9GB of raw data) was reduced to approximately 53 million reads through trimming and filtering. These remaining reads were assembled into 96,491 contigs (Additional file 1). The GC content per contig was $52 \%$ for both the pancreas and liver samples and $49 \%$ for the hypothalamus and bursa/bone marrow samples.

The average length of the assembled contigs was 866 bases with a N50 of 1630 nucleotides. Over 45,000 of these assembled contigs ranged from 200-399 base pairs (Figure 1). The large number of short contigs appears to partially be the result of single end sequencing and

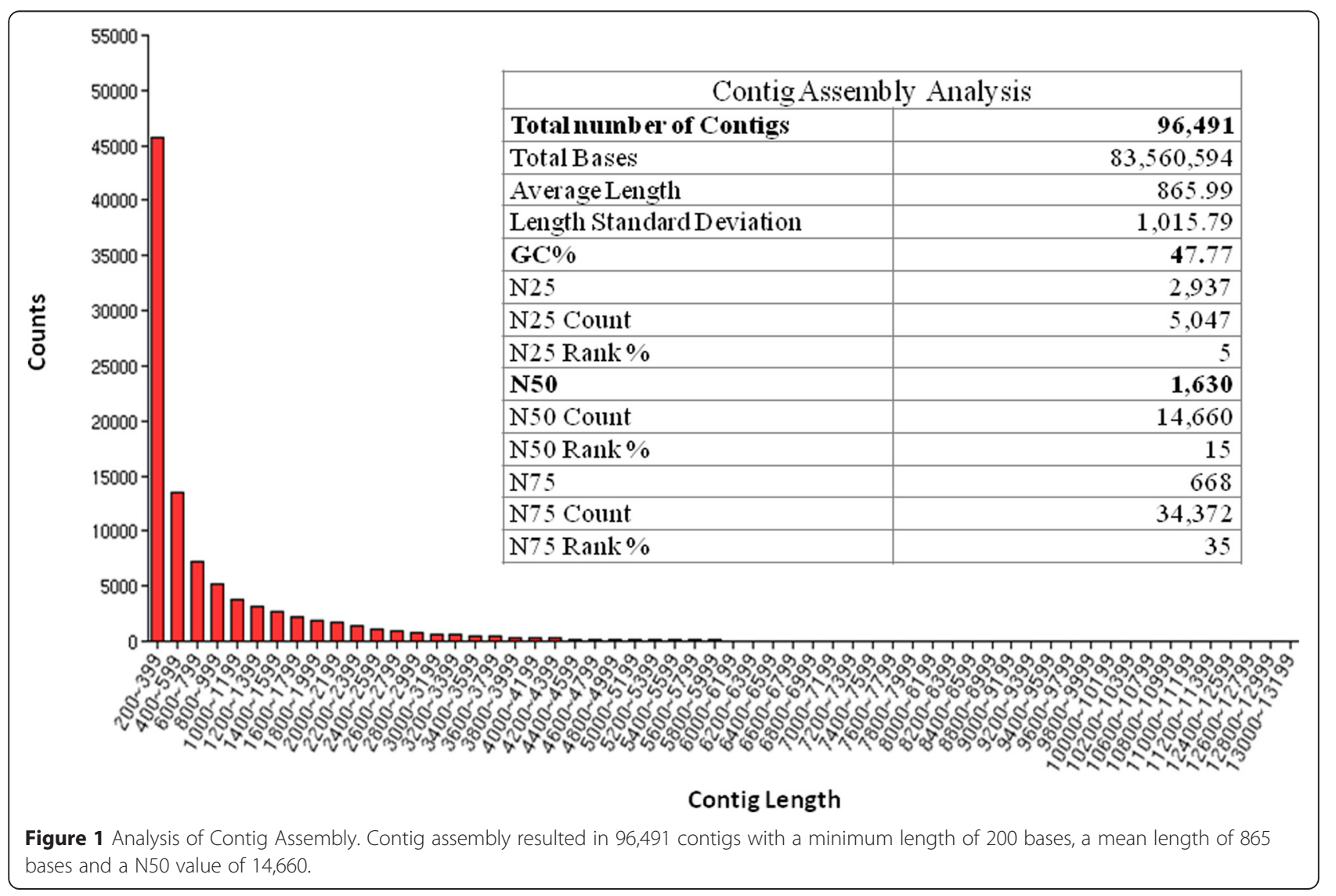


partially due to the assembly method. While Trinity tends to recover more correct transcripts overall than other methods, it also tends to recover a great deal of partial transcript sequences [22].

\section{Functional annotation}

A total of 47,079 contigs assembled by Trinity returned statistically significant $\left(1.0 \mathrm{e}^{-5}\right)$ hits after blastp and BlastX searches against the SwissProt section of UniProt and the entire Chicken and Turkey proteomes.

The Gene Ontology terms assigned to the contigs were well distributed between the categories of biological process, cellular component and molecular function, with a mean level of 6 (Figures 2 and 3). Of these, 38,673 were assigned at least one Gene Ontology term. In addition 3,354 were assigned an enzyme annotation (Figure 4). Transcripts that had e-values rising above $1 \mathrm{e}^{-10}$ were annotated. This Transcriptome Shotgun Assembly project has been deposited at DDBJ/EMBL/GenBank under the accession GBYG00000000. The version described in this paper is the first version, GBYG01000000.

Gene coverage analysis for the annotated sequences revealed that 5,796 or $23.5 \%$ of the sequences that yielded hits covered more than $50 \%$ of the gene to which they shared homology. A total of 866 sequences showed $100 \%$ gene coverage. The predicted open reading frames encoded by the sequences analyzed ranged from 200 to 4000 bases in length of with the majority falling between 100 to 200 bases. Due to the low coverage of the reads and lack of replicates a differential expression analysis between the individual tissues was not performed. FastAnnotator identified 24,349 domains in the query nucleotide sequences with coverage greater than $50 \%$ by searching against domain models from the Conserved Domains Database at an expectation value (e-value) limit of 0.01 (Figure 4).

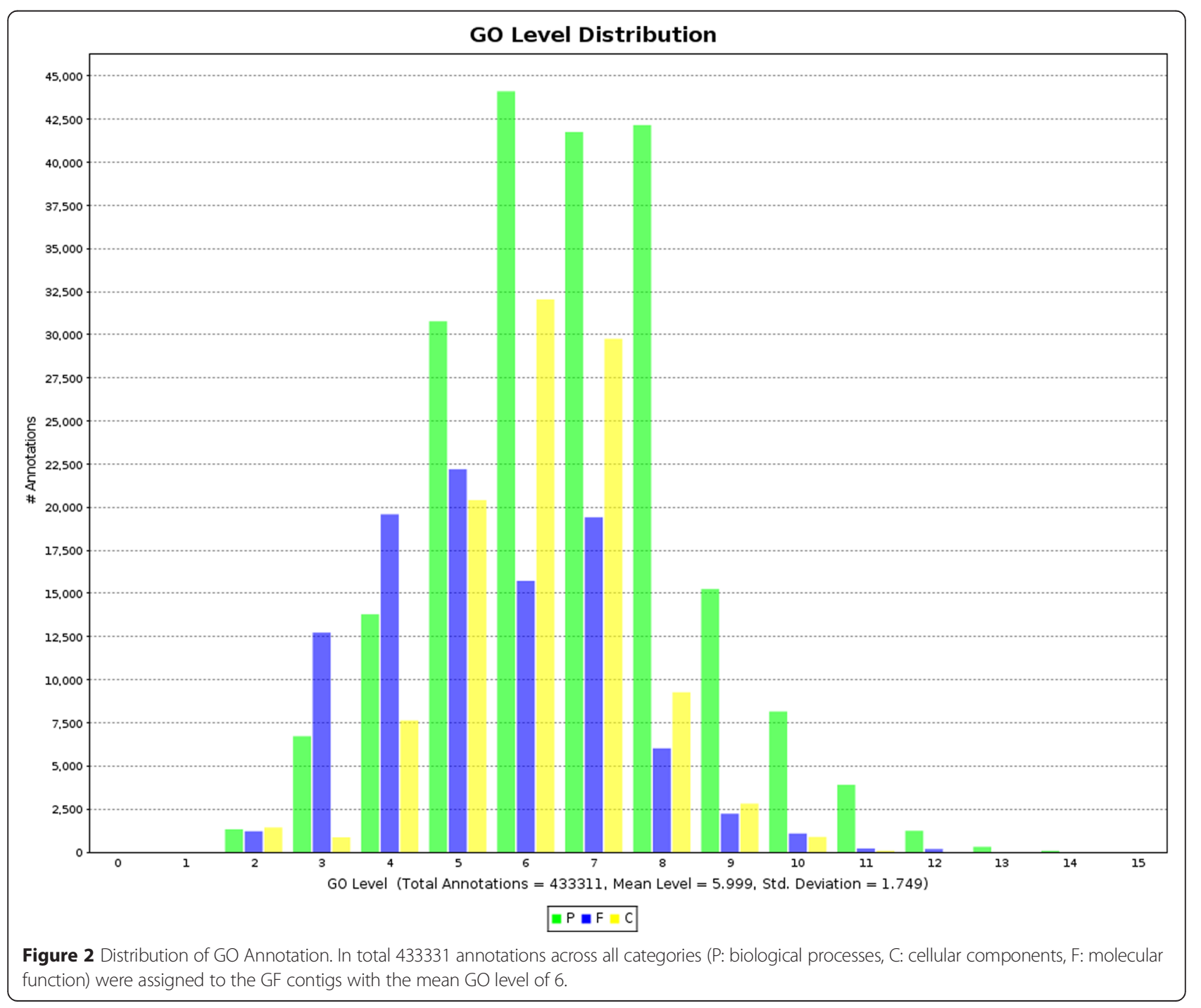




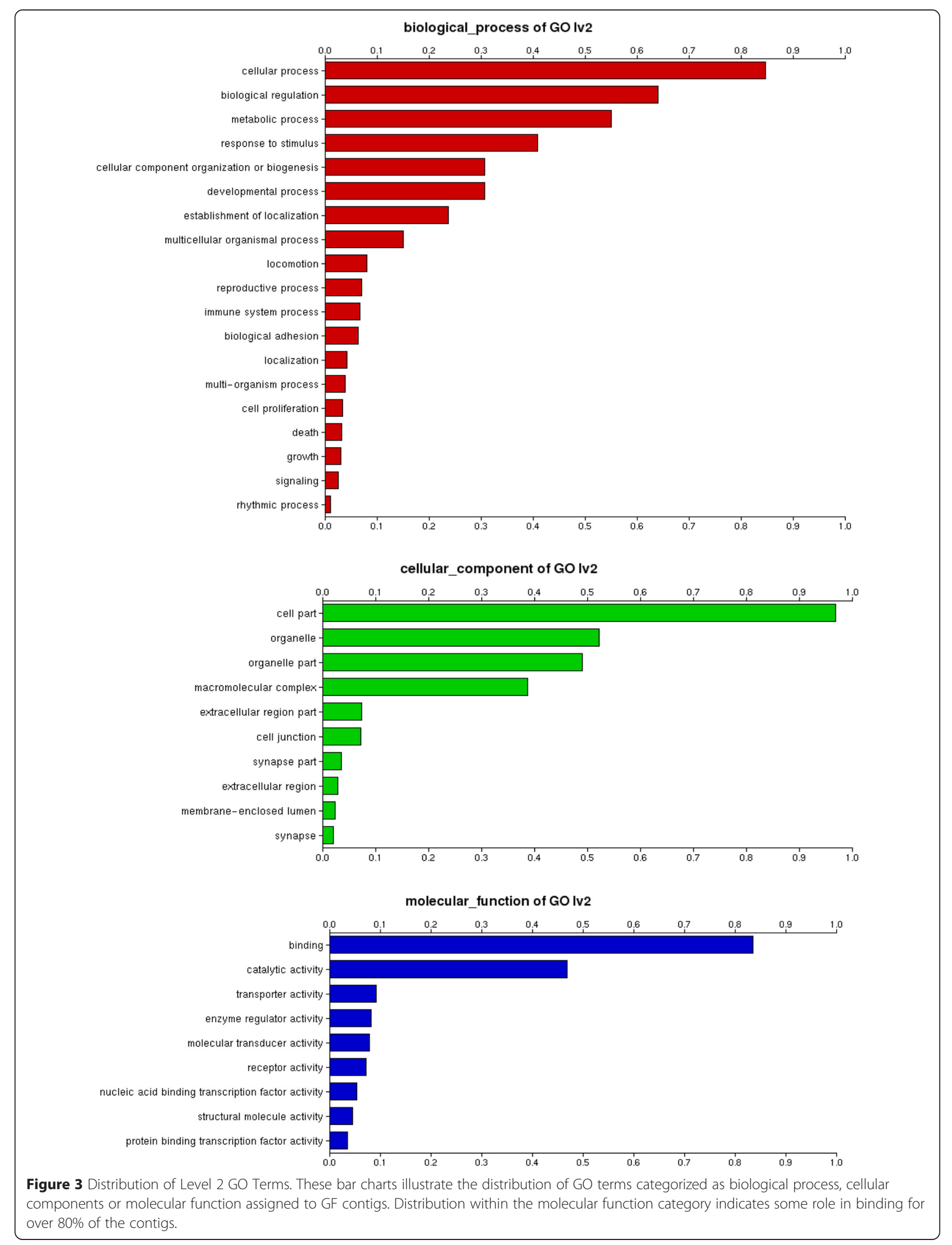




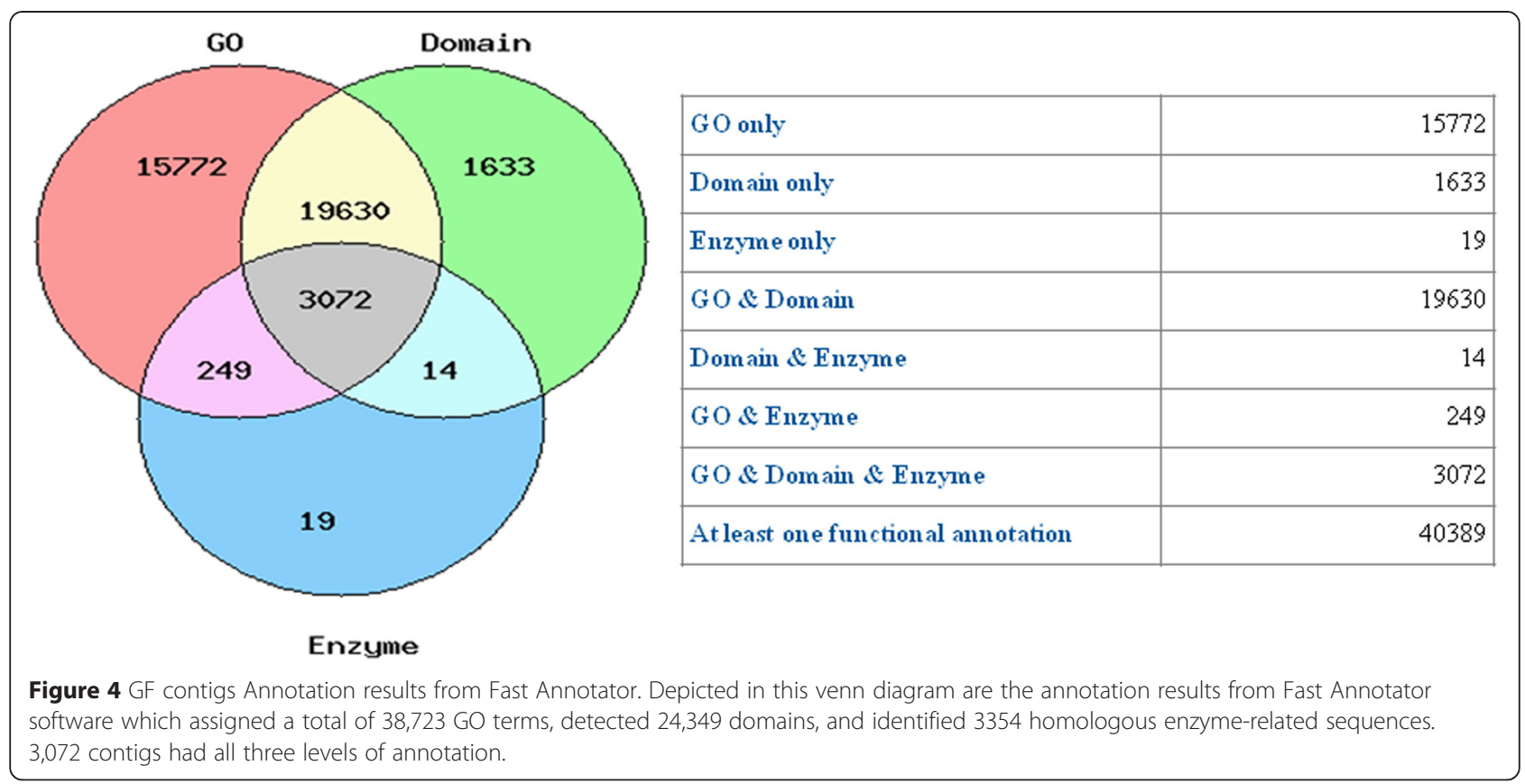

\section{Identification of orthologus contigs}

Forty percent of the contigs were homologous with protein sequences deposited in the blastp database for chicken $(39,376)$ and turkey $(39,474)$ (Additional file 2). GO terms were used to mine the Trinotate output (Additional file 3) and identify contigs annotated with metabolism or immune function. In total 416 transcripts with metabolic function and 703 with immune function were uncovered (Tables 1 and 2).

\section{Conclusion}

Most of the desired traits in farm animals such as body mass, production yield, and disease resistance are quantitative. Over the centuries traditional selective breeding of superior individuals has resulted in the marked enhancement of production traits based on phenotypic expression of desirable traits [23]. These traditional means of breed improvement through phenotypic selection have

Table 1 Metabolic function related GO term search of Trinotate output

\begin{tabular}{lll}
\hline Metabolic Function Terms & Contigs \\
\hline GO:0005976 & polysaccharide metabolic process & 4 \\
GO:0006109 & regulation of carbohydrate metabolic process & 2 \\
GO:0006629 & lipid metabolic process & 252 \\
GO:0008152 & metabolic process & 151 \\
GO:0016052 & carbohydrate catabolic process & 6 \\
GO:0019538 & protein metabolic process & 4 \\
GO:0044262 & cellular carbohydrate metabolic process & 13 \\
GO:0051246 & regulation of protein metabolic process & 13 \\
\hline
\end{tabular}

led to enhancement of economically important traits in cattle, sheep, pigs, poultry and other livestock [24]. One drawback however, is that traditional methods of breeding are limited in their ability to select for traits that are difficult to measure such as fertility, longevity and disease resistance. These traits do not lend themselves to such dramatic improvement through selection alone [23]. It is in these areas that transcriptomic data has the most potential for direct and immediate application.

By focusing on those genes that code for proteins related to traits of interest, poultry scientists have gleaned and applied genetic information to increase the production and performance of chicken and other poultry birds. The transcriptomic data set presented in this study contributes to the genomic and proteomic resources available for GF development. These genetic tools will

Table 2 Immune function related GO term search of Trinotate output

\begin{tabular}{lll}
\hline Immune Function Terms & Contigs \\
\hline GO:0045087 & innate immune response & 445 \\
GO:0006959 & humoral immune response & 37 \\
GO:0006955 & immune response & 244 \\
GO:0050776 & regulation of immune response & 36 \\
GO:0002682 & regulation of immune system & 7 \\
GO:0050778 & positive regulation of immune response & 8 \\
GO:0033025 & regulation of mast cell apoptotic & 4 \\
GO:0045637 & regulation of myelo cell differentiation & 3 \\
GO:0002683 & negative regulation of immune system & 1 \\
GO:0034121 & regulation of protein metabolic process & 2 \\
\hline
\end{tabular}


support the progression of molecular approaches to improve the profitability of guinea fowl production. Our results show that sixty percent of the contigs were non-homologous with protein sequences deposited in the blastp database for chicken. The continued curation of this putative set of novel GF genes is essential for downstream comparative analysis, expression profiling, functional studies and trait selection across avian species.

\section{Methods}

\section{Animals and RNA preparation}

The pancreas, liver, hypothalamus, bone marrow and bursa were harvested from an eight week old male guinea fowl which was housed under a 12-hour light/dark cycle and fed a diet comprising of $3,340 \mathrm{kcal}$ of metabolizable energy $/ \mathrm{kg}$ of diet and $23 \%$ crude protein. Feed and water were provided for ad libitum consumption. Animal use for this study was approved by Tennessee State University Institutional Animal Care and Use Committee (IACUC). Following sacrifice by cervical dislocation, liver (approximately $5 \mathrm{~g}$ from the mid-portion of the anterior sub-segment of the right lobe), pancreas (approximately 2 grams of tissue from the duodenal loop), tibial bone marrow, and bursa (whole organ) were removed and submerged in an RNA stabilization solution ( $\mathrm{pH}$ 5.2) containing $0.5 \mathrm{M}$ EDTA, $1 \mathrm{M}$ sodium citrate, and $700 \mathrm{~g}$ ammonium sulfate dissolved in ultrapure water overnight at $4^{\circ} \mathrm{C}$. Whole heads were flash-frozen in liquid nitrogen. Subsequently, hypothalami were excised by microdissection and submerged in an RNA stabilization solution. All tissue samples were stored at $-80^{\circ} \mathrm{C}$ until use. Total RNA was isolated from each tissue using Qiagen's RNeasy ${ }^{\circ}$ Mini Kit according to the manufacturer's protocol. (Qiagen, Valencia, CA) Total RNA concentrations were determined via NanodropTM Spectrophotometer (Thermo Scientific; Wilmington, DE). Each sample was diluted to $50 \mathrm{ng} / \mu \mathrm{l}$, separated into $50 \mu \mathrm{l}$ aliquots and immediately frozen at $-80^{\circ} \mathrm{C}$. Sample quality was evaluated by visual inspection of a $1 \%$ formaldehyde gel ran at 100 volts for 1 hour. Gel images were captured using the Kodak Gel Logic 1500 Imaging System (Kodak; Rochester, NY). Experion ${ }^{\text {Tw }}$ Automated Electrophoresis System (Bio-Rad; Hercules, CA) was used to confirm RNA quality according to the manufacture's guidelines. Sample quality was also confirmed using a BioAnalyzer (Agilent; Santa Clara, CA). The resulting RIN values for the hypothalamus, pancreas, liver and bursa/bone marrow samples were 9.5, 7.9, 5.3 and 5.5 respectively.

\section{Library construction and iillumina sequencing}

The cDNA library construction was conducted at the Vanderbilt University's Genomic Sciences Resource Center (VUGSR), Nashville, TN (VUGSR). During library construction, mRNA was isolated from $100 \mathrm{ng}$ of total
RNA followed by fragmentation, $1^{\text {st }}$ then $2^{\text {nd }}$ strand cDNA synthesis. The cDNA was end-repaired, size selected and then ligated to adapter sequences. The cDNA libraries were multiplexed and sequenced in one lane using Illumina's Hi-Seq 2000 (Illumina, Inc., San Diego, CA) single end read sequencing platform. The sequencing run produced approximately 74 million single end reads with average length of $101 \mathrm{bp}$. The resulting reads were de-multiplex and reported as separate runs and deposited in the National Institutes of Health (NIH) Short Read Archive (http://ncbi.nlm.nih.gov/sra) (Pancreas: SRS584523, Hypothalamus: SRS413447, Liver: SRS585609, Bone Marrow/Bursa: SRS586251).

\section{Assembly, annotation, and gene ontology analysis}

Prior to assembly, all reads were run through quality control procedures to ensure that Illumina adapters were removed and that only high quality data was used in the assembly. The FastQC program was used to perform an examination of the reads. Based on those results, tools in the fastx toolkit were used to remove Illumina adapters, performing end trimming of reads, as well as filtering reads out of the dataset that had average quality values $<30$ (sup. Figure 1). After these trimming and filtering procedures, approximately 54 million reads remained for assembly.

Assembly and annotation was performed on Blacklight, a SGI UV 1000 cc-NUMA shared-memory system available to U.S. academic researchers through the NSF XSEDE program (www.xsede.org).

Transcripts were assembled de novo using Trinity (r2012-08-14) (available at http://trinityrnaseq.source forge.net/) using the default settings [22,25]. To ensure a uniform transcriptome reference across the datasets, all reads were pooled for assembly then the datasets were individually aligned back to the reference transcriptome.

The transcriptome produced was annotated using Trinotate (r2013-08-26) (available at http://trinotate.source forge.net). The Trinotate suite provides for the functional annotation of de novo assembled transcriptomes and makes use of several annotation techniques including blastp/blastx database searches against reference sequence databases, PFAM domain searches, and various signal predictions. Trinotate integrates this initial annotation information into a relational database that includes reference information from Uniprot, and eggNOG/GO Pathways databases. Due to the modular design of the system and the use of an SQL database, the system was modified to include information contained within the complete proteome sequences of both the chicken and turkey.

The assembled transcripts were also submitted to FastAnnotator for comparative annotation and identification of domains and potential enzyme functions. 
Fast Annotator (available at fastannotator.cgu.edu.tw) was used to analyze the distribution of gene ontology terms, develop graphical representations of the data set and for enzyme identification [26]. Gene Ontonlogy (GO) terms used to identify associated with metabolic functions: GO:0005976, GO:0006109, GO:0006629, GO:0008152, GO:0016052, GO:0019538, GO:0044262,GO:0051246. GO terms used to identify associated with immune functions: GO:0045087, GO:0006959, GO:0050776, GO:0002682, GO:0050778, GO:0033025, GO:0045637, GO:0002683, GO:0034121.

\section{Availability of supporting data}

The data sets supporting the results of this article are included within the article and its supplemental files.

\section{Additional files}

Additional file 1: GF_Trinity Output Fasta.

Additional file 2: Blastp XLS.

Additional file 3: Trinotate Annotation Report.

\section{Abbreviations}

cDNA: Complimentary Deoxy-Ribose Nucleic Acid; GC: Guanine Cytosine; GF: Guinea Fowl; GO: Gene Ontology; mRNA: messenger Ribose nucleic acid; PFAM: The Pfam database is a large collection of protein families; RNA: Ribose Nucleic Acid; RNA-seq: Ribose Nucleic Acid Sequencing; SQL: Structured Query Language.

\section{Competing interests}

The authors declare that they have no competing interests.

\section{Authors' contributions}

$C D$, JT, and GK performed sample collection and processing. CD, AR and HN participated in the transcriptome assembly and anlysis of bioinformatic data. $C D, A R, H N, X W$ and $S N$ participated in the design of the study. CD, AR, XWand SN conceived of the study, and participated in its design and coordination and helped to draft the manuscript. All authors read and approved the final manuscript.

\section{Acknowledgements \\ Special thanks to Darren Wright, Angela Washington, Tiffany Johnson, Kiimya Nelson, Joseph Donkor, Boniface Kimathi, Harsha Subramanya for their technical expertise. \\ This work was supported by the National Institutes of Health, National Institute of General Medical Sciences Minority Access to Research Careers Grant T36-GM-095335 to the Pittsburgh Supercomputing Center. This work used Blacklight at the Pittsburgh Supercomputing Center, part of the Extreme Science and Engineering Discovery Environment (XSEDE), which is supported by National Science Foundation grant number ACl-1053575. Any opinions, findings, and conclusions or recommendations expressed in this material are those of the author(s) and do not necessarily reflect the views of the National Science Foundation.}

\section{Author details}

${ }^{1}$ College of Agriculture, Human and Natural Sciences, Tennessee State University, Nashville, Tennessee, USA. ${ }^{2}$ Pittsburgh Supercomputing Center, Carnegie Mellon University, Pittsburgh, Pennsylvania, USA.

Received: 6 January 2015 Accepted: 10 April 2015

Published online: 07 May 2015

\section{References}

1. Nahashon SN, Amenyenu A, Muhammad S, Adefope N, and Wright D: Assessment of awareness and constraints in production of guinea fowl in Tennessee and other parts of the United States. 2004. Southern Animal Science Association Abstracts pp. 7.

2. Collins $\perp B P$, Voelckel C, Joly S. An approach to transcriptome analysis of non-model organisms using short-read sequences. Genome Informatics. 2008;21:3-14.

3. KÜNstner A, Wolf JBW, BackstrÖM N, Whitney O, Balakrishnan CN, Day L, et al. Comparative genomics based on massive parallel transcriptome sequencing reveals patterns of substitution and selection across 10 bird species. Mol Ecol. 2010;19:266-76.

4. Chu JHLR, Yeh CF, Hsu YC, Li SH. Characterization of the transcriptome of an ecologically important avian species, the Vinous-throated Parrotbill Paradoxornis webbianus bulomachus (Paradoxornithidae; Aves). BMC Genomics. 2012;13(149):1-11.

5. Baeza E, Juin H, Rebours G, Constantin P, Marche G, Leterrier C. Effect of genotype, sex and rearing temperature on carcase and meat quality of guinea fowl. Br Poult Sci. 2001;42(4):470-6.

6. Barna J, Wishart GJ. Excess nuclear DNA in spermatozoa of guinea fowl. Theriogenology. 2003;59(8):1685-91.

7. Cattoli G, De Battisti C, Toffan A, Salviato A, Lavazza A, Cerioli M, et al. Co-circulation of distinct genetic lineages of astroviruses in turkeys and guinea fowl. Arch Virol. 2007;152(3):595-602.

8. Claudius L, Yoshimi Y, Yoichiro H, Rudovick K, Koichi M. Molecular cloning, expression profile and functional implications of clusterin in the pituitary gland of helmeted guinea fowl (Numida meleagris). Cell Biol Int. 2005;29(8):675-86.

9. Girish PS, Anjaneyulu ASR, Viswas KN, Santhosh FH, Bhilegaonkar KN, Agarwal RK, et al. Polymerase Chain Reaction-Restriction Fragment Length Polymorphism of Mitochondrial 125 rRNA Gene: A Simple Method for Identification of Poultry Meat Species. Vet Res Commun. 2007;31(4):447-55.

10. Kayang BB, Inoue-Murayama M, Hoshi T, Matsuo K, Takahashi H, Minezawa $\mathrm{M}$, et al. Microsatellite loci in Japanese quail and cross-species amplification in chicken and quinea fowl. GSE. 2002;34(2):233-53.

11. Luziga C, Usui M, Yoichiro H, Kazwala R, Yamamoto Y, Mamba K. Gene expression and immunohistochemical localization of megalin in the anterior pituitary gland of helmeted guinea fowl (Numida meleagris). J Mol Histol. 2007;38(1):65-77.

12. Ruan W, Wu Y, Zheng SJ. Different genetic patterns in avian Toll-like receptor (TLR)5 genes. Mol Biol Rep. 2012;39(4):3419-26.

13. Saxena VK, Singh H, Pal SK, Kumar S. Genetic studies on primary antibody response to sheep erythrocytes in guinea fowl. Br Poult Sci. 1997;38(2):156-8.

14. Sharma D, Rao KB, Singh HP, Totey SM. Randomly amplified polymorphic DNA (RAPD) for evaluating genetic relationships among varieties of guinea fowl. Genetic Analysis. 1998;14(4):125-8.

15. Shibusawa M, Nishida-Umehara C, Masabanda J, Griffin DK, Isobe T, Matsuda Y. Chromosome rearrangements between chicken and guinea fowl defined by comparative chromosome painting and FISH mapping of DNA clones. Cytogenetic Genome Res. 2002;98(2-3):225-30.

16. Smith EJ, Shi L, Prevost L, Drummond P, Ramlal S, Smith G, et al. Expressed Sequence Tags for the Chicken Genome from a Normalized, Ten-Day-Old White Leghorn Whole Embryo cDNA Library. 2. Comparative DNA Sequence Analysis of Guinea Fowl, Quail, and Turkey Genomes. Poult Sci. 2001;80(9):1263-72.

17. Vidal O, Araguas RM, Fernandez E, Heras S, Sanz N, Pla C. Melanism in guinea fowl (Numida meleagris) is associated with a deletion of Phenylalanine-256 in the MC1R gene. Anim Genet. 2010;41(6):656-8.

18. van der Most PJ, de Jong B, Parmentier HK, Verhulst S. Trade-off between growth and immune function: a meta-analysis of selection experiments. Funct Ecol. 2011;25(1):74-80.

19. Greer AW. Trade-offs and benefits: implications of promoting a strong immunity to gastrointestinal parasites in sheep. Parasite Immunology. 2008;30(2):123-32.

20. Knowles SCL, Nakagawa S, Sheldon BC. Elevated reproductive effort increases blood parasitaemia and decreases immune function in birds: a meta-regression approach. Funct Ecol. 2009;23(2):405-15.

21. Rantala MJ, Roff DA. An Analysis of Trade-offs in Immune Function, Body Size and Development Time in the Mediterranean Field Cricket, Gryllus bimaculatus. Functional Ecol. 2005;19(2):323-30. 
22. Grabherr MG, Haas BJ, Yassour M, Levin JZ, Thompson DA, Amit I, et al. Regev, Aviv: Full-length transcriptome assembly from RNA-Seq data without a reference genome. Nat Biotech. 2011;29(7):644-52.

23. Eggen A. The development and application of genomic selection as a new breeding paradigm. Animal Frontiers. 2012;2(1):10-5.

24. Raj GD: Improvement of Farm Animal Breeding by DNA Sequencing. DNA Sequencing-Methods and applications 2012:85.

25. Haas BJ, Papanicolaou A, Yassour M, Grabherr M, Blood PD, Bowden J, et al. De novo transcript sequence reconstruction from RNA-seq using the Trinity platform for reference generation and analysis. Nat Protocols. 2013;8(8):1494-512.

26. Chen T-W, Gan R-C, Wu T, Huang P-J, Lee C-Y, Chen Y-Y, et al.

FastAnnotator- an efficient transcript annotation web tool. BMC Genomics. 2012;13 Suppl 7:59.

\section{Submit your next manuscript to BioMed Central and take full advantage of:}

- Convenient online submission

- Thorough peer review

- No space constraints or color figure charges

- Immediate publication on acceptance

- Inclusion in PubMed, CAS, Scopus and Google Scholar

- Research which is freely available for redistribution 